\title{
Comparative Evaluation of $0.2 \%$ Chlorhexidine Mouth Rinse with and without an Antidiscoloration System: A Clinical Study
}

\author{
${ }^{1}$ Richard Pereira, ${ }^{2}$ Sidheshwar G Phad
}

\begin{abstract}
Introduction: Chlorhexidine ( $\mathrm{CHX})$ is considered the gold standard agent for its clinical efficacy in chemical plaque control. The addition of other active ingredients may even reduce the antiplaque effectiveness. New mouthwash containing $\mathrm{CHX}$ with addition of an antidiscoloration system (ADS), i.e., Plasdone $\mathrm{K}-29 / 32$, that claims to bind to stains, increases their solubility in water and effects their removal; thus the mouthwash promises not only to prevent plaque formation but also to avoid staining.
\end{abstract}

Aim: To clinically evaluate the efficacy of a $0.2 \% \mathrm{CHX}$ mouth rinse with and without an ADS.

Materials and methods: The present study was a tripleblinded, prospective, cohort, and crossover study. This study consisted of 15 subjects. Supragingival scaling and polishing were done and then subjects were asked to rinse with a randomly allocated mouthwash for 15 days. Subjects used either mouthwash A $(0.2 \% \mathrm{CHX}$ with $\mathrm{ADS})$ or mouthwash $\mathrm{B}(0.2 \%$ $\mathrm{CHX}$ ). After 15 days, the discoloration index and plaque index (PI) were recorded. After 15 days washout period, on the 30th day from baseline, supragingival scaling and polishing were carried out, and each subject used the subsequent mouthwash. On 45th day from baseline, the discoloration and PIs were recorded and comparisons were made.

Results: No significant difference in the PI between groups I and II mouthwash was seen $(p \geq 0.05)$, whereas significant difference was found in the discoloration index between groups I and II mouthwash $(p \leq 0.05)$.

Conclusion: The addition of Plasdone does not reduce the efficacy of $\mathrm{CHX}$ mouthwash. The current study also proved that $\mathrm{CHX}$ with ADS caused less staining than $\mathrm{CHX}$ alone.

Keywords: Antidiscoloration system, Chlorhexidine, Mouthwashes, Plasdone, Staining.

How to cite this article: Pereira R, Phad SG. Comparative Evaluation of $0.2 \%$ Chlorhexidine Mouth Rinse with and without an Antidiscoloration System: A Clinical Study. J Contemp Dent 2017;7(1):53-56.

Source of support: Nil

Conflict of interest: None

\footnotetext{
${ }^{1}$ Professor, ${ }^{2}$ Postgraduate Student

1,2Department of Periodontics, Mahatma Gandhi Mission's Dental College and Hospital, Navi Mumbai, Maharashtra, India

Corresponding Author: Sidheshwar G Phad, Postgraduate Student, Department of Periodontics, Mahatma Gandhi Mission's Dental College and Hospital, Navi Mumbai, Maharashtra, India Phone: +919922337010, e-mail: sphad33@gmail.com
}

\section{INTRODUCTION}

Bacterial plaque plays an essential role in the development of periodontal disease since its accumulation inevitably leads to gingivitis. ${ }^{1}$ Oral health can be improved through effective plaque control; mechanical methods fail to give optimal results for oral health because the techniques are not done consistently or thoroughly. Studies have also demonstrated that daily toothbrushing and flossing are not practiced consistently and are not done for adequate amount of time to thoroughly remove the plaque. Because of these drawbacks on home oral care practices, other methods of oral care are required. ${ }^{2}$

A chemical approach has been introduced to deal with the potential deficiencies of daily self-performed oral hygiene. ${ }^{3}$ The challenge with chemical plaque control is to develop an active antiplaque agent that does not disturb the natural flora of the oral cavity. Antibiotics could be a better idea for the control and growth of microorganisms, but use of antibiotics has several limitations, such as risks of bacterial resistance, hypersensitivity reactions, or superinfections. ${ }^{4}$ Because of its established use and efficacy, chlorhexidine (CHX) is the gold standard agent when compared with other chemical factor efficacies. Chlorhexidine is the effective antibacterial agent for chemical plaque control and it is extensively used for reduction of plaque and gingivitis by $60 \% .{ }^{5}$ Chlorhexidine is adsorbed rapidly on dental surfaces, mucous membranes, and salivary proteins and is released gradually over 8 to 12 hours. ${ }^{6}$ This property, called substantivity, affords a convenient dose schedule for patients every 12 hours. But the use of $\mathrm{CHX}$ is burdened by some side effects that could affect patient compliance. ${ }^{7}$ The most notable of these is the staining it produces. In recent years, various $\mathrm{CHX}$ solutions have come onto the market promising better tolerance with the same efficacy. The addition of other active ingredients in mouthwash does not produce beneficial effects, but they may reduce the antiplaque effectiveness or increase tongue staining. ${ }^{8}$ Chlorhexidine was superior in inhibiting plaque and reducing bacterial growth when compared with the solution with anti-discoloration system (ADS). ${ }^{9}$ There is a possibility that by the addition of other chemical agents to CHX mouthwashes in an attempt to improve its effects, 
it may result in the inactivation of the antiseptic property due to the high cationic value of its molecule.

A new mouthwash, which contains CHX and additionally an ADS), promises not only to prevent plaque formation but also to avoid staining.

\section{MATERIALS AND METHODS}

Ethical clearance was obtained from the Institutional Ethical Committee of Mahatma Gandhi Mission's Dental College and Hospital. This study was conducted within the institute and restricted only to healthy dental cohorts. They enrolled after being explained about the purpose and about the products to be used in the study with possible side effects. Healthy dental student volunteers aged between 18 and 25 years consented to their participation in the study by a signed informed consent form and were enrolled for the study once they fulfilled the inclusion criteria.

The present study was a triple-blinded, prospective, cohort and crossover study. The study population consisted of 15 subjects. The subject's selection criteria included presence of minimum 20 natural scorable teeth of permanent dentition excluding third molars, systemically healthy individuals, presenting good level of oral hygiene evaluated based on a mean plaque index (PI) score $<1,{ }^{10}$ and subjects who were willing to abstain from any other means of oral hygiene maintenance throughout the investigation period.

Subjects were excluded if showing any signs of plaque-induced gingival or periodontal disease, received any form of periodontal therapy, surgical or nonsurgical treatment within past 6 months, antibiotic and/or anti-inflammatory therapy within the past 3 months, orthodontic or prosthetic appliances, untreated grossly carious teeth, known history of systemic disease, and physically debilitated subjects. History of known allergies to constituents was found in the mouthwash assigned during the investigation.

\section{Division of Groups}

Mouthwash A: Commercially available 0.2\% CHX with Plasdone K-29/32 ADS

Mouthwash B: Commercially available $0.2 \%$ CHX mouthwash

\section{Method of Blinding}

The mouthwashes were dispensed in identical radiopaque white bottles labeled as mouthwash A and mouthwash B.

At baseline, a detailed case history was recorded to include the subjects according to the inclusion criteria. Complete oral prophylaxis using hand and ultrasonic scaler was done, following which each subject used a randomly assigned one of two mouthwashes (mouthwash A or B) for 15 days. Subjects were asked to follow the following instructions, which were explained to them verbally along with a written reminder, and use the assigned mouthwash to rinse the mouth vigorously with $10 \mathrm{~mL}$ solution twice daily for 1 minute using a stopwatch and then spit it out. Subjects were asked not to eat or drink for 30 minutes after mouth rinsing and maintain the rinsing records and record any unexpected occurrence in the diary provided.

At the second visit (15 days after baseline), discoloration and PIs were recorded. Complete oral prophylaxis using hand and ultrasonic scaler was done. Subjects were asked to resume their normal methods of oral hygiene maintenance. During the study period, modified Bass brushing technique was advised. Then 15 days washout period followed. At the third visit (30 days from baseline), complete oral prophylaxis was done. Subsequently, each subject used second mouthwash for 15 days. Same instructions were given to subjects during crossover period; subjects were recalled for fourth visit (45 days after baseline), discoloration and PIs were recorded. Complete oral prophylaxis using hand and ultrasonic scaler was done. Subjects were asked to resume their previous methods of oral hygiene practices.

\section{Statistical Analysis}

The data were analyzed and presented using descriptive statistics. Paired t-test was used for the intergroup comparison between the groups; $5 \%$ level of significance was used for the analysis. The p-value $<0.05$ was treated as significant.

\section{RESULTS}

All subjects completed the study. No subject reported any complication or unexpected complaints. When the groups were compared for efficacy using the PI, no significant difference was seen in the PI between groups I and II mouthwash ( $\mathrm{p}$-value for the t-test is $>0.05$ ).

Graph 1 shows PI ${ }^{10}$ over time. The two mouthwashes were equally effective in reducing plaque in the patient. No statistically significant differences were observed in plaque reduction ( $p=0.1029$ ) between the two mouthwashes (Table 1). When the groups were compared using the discoloration index, both caused staining but the difference was significant in that mouthwash A caused lesser staining.

With mouthwash $B$, discoloration index ${ }^{11}$ is much higher than with mouthwash A (Figs $1 \mathrm{~A}$ and B). The values obtained are statistically significant $(p \leq 0.05)$. 


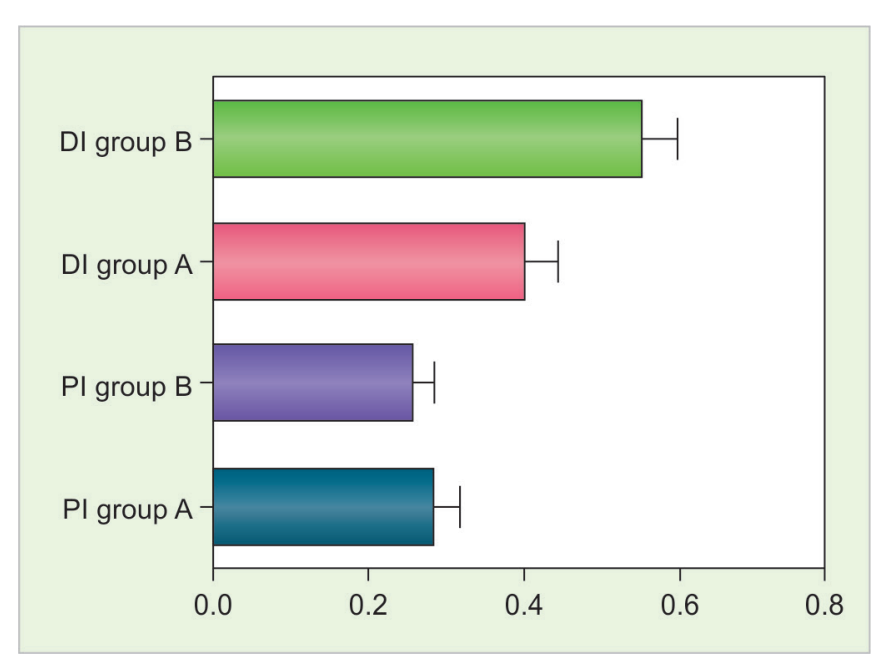

Graph 1: Discoloration index and PI over time

\section{DISCUSSION}

Chlorhexidine is considered as a gold standard in chemical plaque control regimen, but has the noted side effect of causing discoloration over prolonged use. Recently, active ingredients are being added to the mouthwashes to improve their effectiveness. According to Bascones et $\mathrm{al}^{8}$ the addition of other active ingredients does not produce beneficial effects, but may even reduce the antiplaque effectiveness or increase tongue staining. $\mathrm{Li}$ et $\mathrm{al}^{11}$ in their experimental gingivitis model concluded that though CHX with an ADS appeared to be effective in preventing staining of the teeth, the ability of a CHX mouthwash in preventing plaque accumulation and gingivitis was greatly hampered by the addition of ADS. In our clinical study, we evaluated the addition of an ADS Plasdone K-29/32 in CHX, with the main purpose of our study being to assess if adding such active ingredients like Plasdone K-29/32 to CHX altered its effectiveness.

For this purpose, we compared $0.2 \%$ CHX-containing mouthwash with and without addition of ADS. Plasdone $\mathrm{K}-29 / 32$ is supposedly a water-soluble, high molecular weight homopolymer of vinylpyrrolidone that has been designed as an antidiscoloration agent meant as an additive in CHX to reduce discoloration. Plasdone K-29/32 acts by binding to stain-causing chemicals; increases in their solubility result in removal of stains. Plasdone is also nonoxidative as it is neither peroxide based nor a peroxide generator and is nonabrasive.

For study purpose, we designed triple-blinded, prospective, cohort, and crossover study. After analyzing the results obtained during our clinical study comparing

Table 1: Comparison of $\mathrm{PI}$ and discoloration index between the two groups

\begin{tabular}{lcc}
\hline & $P I$ & Discoloration index \\
\hline Mean of differences & -0.0226 & 0.1551 \\
SD of differences & \pm 0.05016 & \pm 0.06327 \\
SEM of differences & 0.01295 & 0.01634 \\
95\% confidence interval & -0.05038 to 0.005177 & 0.12 to 0.1901 \\
Correlation coefficient $(r)$ & -0.3898 & -0.2167 \\
$R^{2}$ (partial eta squared) & 0.1787 & 0.8655 \\
p-value & 0.1029 & $<0.0001$ \\
$t$, df & $\mathrm{t}=1.745 \mathrm{df}=14$ & $\mathrm{t}=9.492 \mathrm{df}=14$ \\
Number of pairs & 15 & 15 \\
\hline
\end{tabular}

SD: Standard deviation; SEM: Standard error of mean
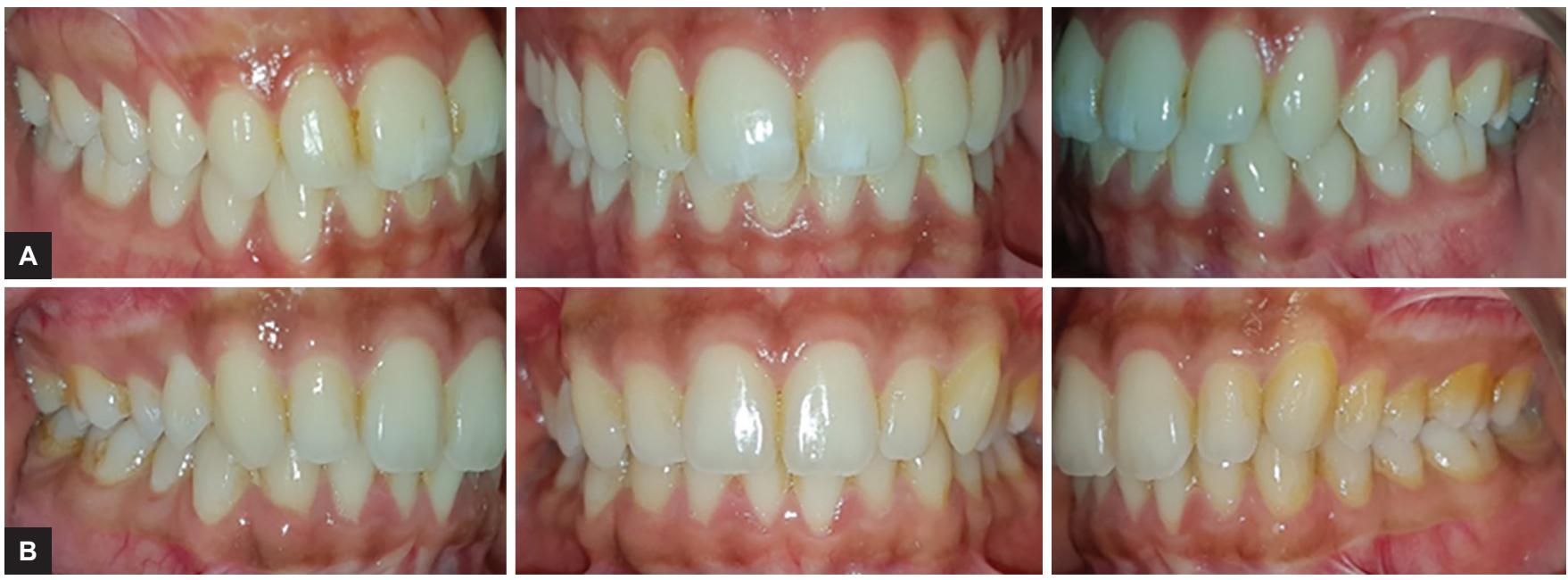

Figs $1 \mathrm{~A}$ and $\mathrm{B}$ : (A) Discoloration due to mouthrinse $\mathrm{A}, 0.2 \% \mathrm{CHX}$ with $\mathrm{ADS}$; and $(\mathrm{B})$ mouthrinse $\mathrm{B}, 0.2 \% \mathrm{CHX}$ 
$0.2 \% \mathrm{CHX}$ mouthwash with ADS to the $0.2 \% \mathrm{CHX}$ alone, we observed that the mouthwash with ADS has similar antiplaque effects. A noticeable decrease in staining was observed with the mouthwash with Plasdone. The present clinical study supports the efficacy of CHX ADS mouthwash showing no differences in terms of plaque accumulation. Bernardi et $\mathrm{a}^{12}$ also found that there was no significant difference in relation to plaque and gingival index between two mouthwashes in healthy patients, but a statistically significant difference was observed in the adverse effect of staining, demonstrating that the mouthwash with ADS prevented staining. Solis et $\mathrm{al}^{2}$ also reported CHX with ADS had less staining than CHX alone during a usage period of 15 days. However, the two mouthwashes seemed to be equally effective as antiplaque and antigingivitis agents. Cortellini et $\mathrm{al}^{13}$ in their triple-blinded study established that $\mathrm{CHX}$ with ADS caused less staining than $\mathrm{CHX}$ alone and is efficacious as $\mathrm{CHX}$ in reducing the plaque and gingival inflammation after periodontal flap surgery. Graziani et $\mathrm{al}^{14}$ in a randomized clinical trial compared CHX with and without ADS, and suggested that there was lighter staining found in the CHX with ADS group.

Addy et $a{ }^{15}$ in their in vitro study did not find statistically significant differences in staining with the ADS added in the 0.12 and $0.2 \% \mathrm{CHX}$ mouthwashes vs $0.2 \%$ $\mathrm{CHX}$ and water as a negative control. Our study also contradicts the findings of Arweiler et $\mathrm{al}^{9}$ who studied $0.2 \%$ CHX mouthwash without alcohol to ADS vs $0.2 \%$ CHX mouthwash with 7\% ethanol to a placebo solution containing sorbitol, concentrated pepper, and alcohol at $14 \%$ where the authors concluded that besides reducing staining, the ADS system also decreased the effectiveness of CHX on dental plaque.

\section{CONCLUSION}

Chlorhexidine mouthwash alone was found to be more effective in plaque control as compared with the mouthwash with Plasdone, though there was minimal difference between the two, thus implying that Plasdone does not reduce the efficacy of CHX mouthwash. The current study also proved that CHX with ADS caused less staining than $\mathrm{CHX}$ alone, thus proving to be an effective solution to the problem of staining caused by $\mathrm{CHX}$ in the long run.

\section{ACKNOWLEDGMENTS}

The authors are grateful to ICPA Pharmaceuticals, Mumbai, for providing the mouthwash samples, for the study, and for their attention and availability provided throughout the research. The authors want to acknowledge all the subjects of the study who voluntarily participated in the study group. Authors also thank the Mahatma Gandhi Mission's Dental College and Hospital for constant support.

\section{REFERENCES}

1. Löe H, Theilade E, Jensen SB. Experimental gingivitis in man. J Periodontol 1965 May;36:177-187.

2. Solıs C, Santos A, Nart J, Violant D. $0.2 \%$ chlorhexidine mouthwash with an antidiscoloration system versus $0.2 \%$ chlorhexidine mouthwash: a prospective clinical comparative study. J Periodontol 2011 Jan;82(1):80-85.

3. Mandel ID. Chemotherapeutic agents for controlling plaque and gingivitis. J Clin Periodontol 1988 Sep;15(8):488-498.

4. Seymour, A.; Heasman, A. Anti-plaque and anti-calculus agents. In: Seymour A.; Heasman A., editors. Drugs, diseases, and the periodontium. New York: Oxford University Press; 1992. p. 151-179.

5. Ciancio, SG.; Nisengard, RJ. Control and prevention of periodontal disease. In: Nisengard RJ.; Newman MG., editors. Oral microbiology and immunology. Philadelphia: WB Saunders; 1994. p. 385-390.

6. Rölla G, Melsen B. On the mechanism of the plaque inhibition by chlorhexidine. J Dent Res 1975 Jun;54(Spec. Issue B):57-62.

7. Ciancio SG. Antiseptics and antibiotics as chemotherapeutic agents for periodontitis management. Compend Contin Educ Dent 2000 Jan;21(1):59-62, 64, 66 passim, quiz 78.

8. Bascones A, Morante A, Mateos L, Mata M, Poblet J. Influence of additional active ingredients on the effectiveness of non-alcoholic chlorhexidine mouthwashes: a randomized controlled trial. J Periodontol 2005 Sep;76(9):1469-1475.

9. Arweiler NB, Boehnke N, Sculean A, Hellwig E, Auschill TM. Differences in efficacy of two commercial $0.2 \%$ chlorhexidine mouthrinse solutions: a 4-day plaque regrowth study. J Clin Periodontol 2006 May;33(5):334-339.

10. Löe H. The gingival index, the plaque index and the retention index systems. J Periodontol 1967 Nov-Dec;38(6):610-616.

11. Li W, Wang RE, Finger M, Lang NP. Evaluation of the antigingivitis effect of a chlorhexidine mouthwash with or without an antidiscoloration system compared to placebo during experimental gingivitis. J Investig Clin Dent 2014 Feb;5(1): 15-22.

12. Bernardi F, Pincelli MR, Carloni S, Gatto MR, Montebugnoli L. Chlorhexidine with an anti-discoloration system. A comparative study. Int J Dent Hyg 2004 Aug;2(3):122-126.

13. CortelliniP,Labriola A,ZambelliR,PratoGP,NieriM,TonettiMS. Chlorhexidine with an anti-discoloration system after periodontal flap surgery: a cross-over, randomized, triple-blind clinical trial. J Clin Periodontol 2008 Jul;35(7):614-620.

14. Graziani F, Gabriele M, D'Aiuto F, Suvan J, Tonelli M, Cei S. Dental plaque, gingival inflammation and tooth discolouration with different commercial formulations of $0.2 \%$ chlorhexidine rinse: a double-blind randomized controlled clinical Trial. Oral Health Prev Dent 2015;13(2):101-111.

15. Addy M, Sharif N, Moran J. A non-staining chlorhexidine mouthwash? Probably not: a study in vitro. Int J Dent Hyg 2005 May;3(2):59-63. 